\title{
P-0353 Clinical verification of accuracy of noninvasive blood glucose monitor based on metabolic heat conformation method
}

Ang Li ${ }^{\mathrm{a}}$, Zhanxiao Geng ${ }^{\mathrm{b}}$, Xiaohui Guo ${ }^{\mathrm{a}}$, Fei Tang ${ }^{\mathrm{b} *}$

a Department of Endocrinology, Peking University First Hospital, Beijing 100034, China

b State Key Laboratory of Precision Measurement Technology and Instruments, Department of Precision Instrument,

Tsinghua University, Beijing 100084, China

Background: As a global chronic disease, diabetes is associated with high morbidity and cannot be fully cured with current technology. In order to achieve better long-term control of glucose, it requires continuous monitoring of metabolic indices. The most commonly used glucose monitor is finger stick testing glucose meters. When using it as a method for frequent glucose monitoring, it can cause physical discomfort, infection risk and contradiction. Noninvasive glucose monitoring can accomplish painless, risk-free, low-cost and frequent testing of glucose, which is an ideal method for self-monitoring of glucose.

Aims: To verify the accuracy of noninvasive blood glucose monitor based on metabolic heat conformation method in patients with type 2 diabetes.

The Noninvasive Glucose Monitor: Based on the theory of metabolic heat conformation method, Tsinghua University and Beijing Sunicare Medical Technologies Co., Ltd. developed a noninvasive glucose monitor. The monitor includes a host and a probe, as shown in Fig. 1. The probe is like a finger clip and the sensors are located inside the probe. The host is like an ipad, which is used for human-computer interaction, control the signal acquisition, data processing and result presenting.

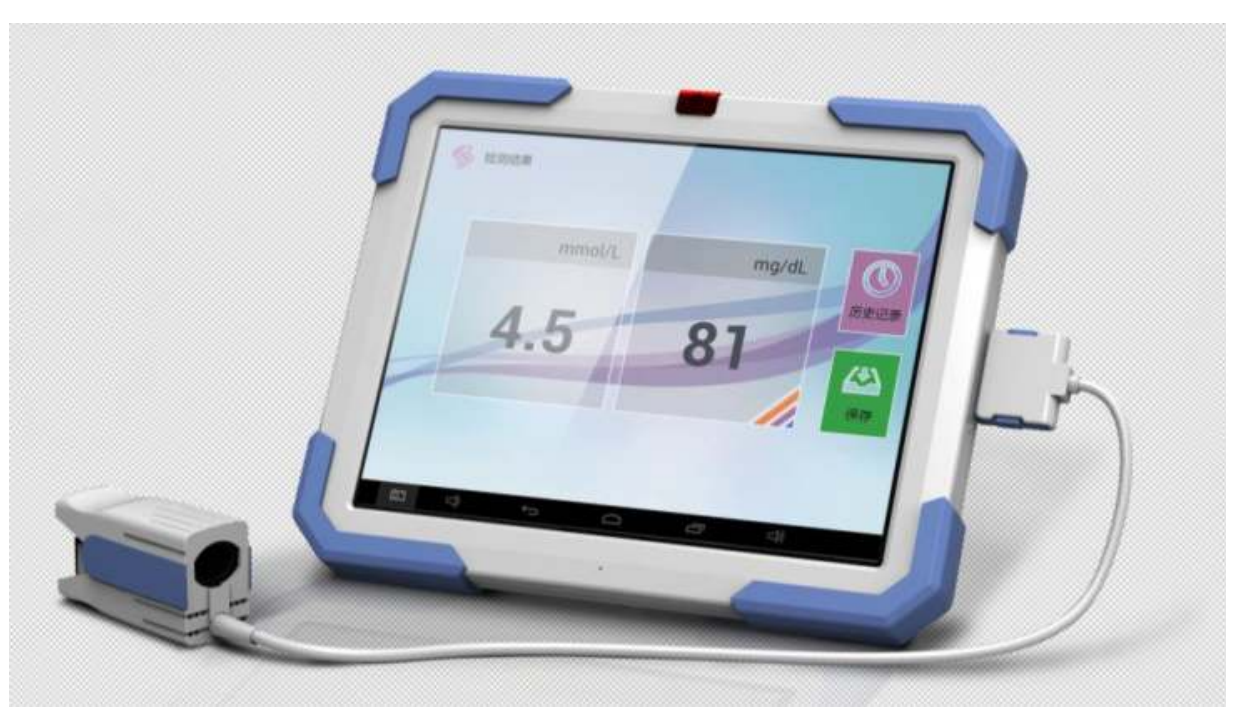

Figure 1 The noninvasive glucose monitor

The Probe: The structure of the probe is shown in Fig. 2. There is a humidity sensor which is used to measure the environment humidity and body humidity, a radiation temperature sensor which is used to measure the environment temperature and body temperature, a heat transfer stick and two resistance thermometers which are used to measure the blood flow, four LEDs (light emitting diode) and a photodiode which are used to measure the $\mathrm{SpO} 2$ (oxygen saturation). When inserting the figure, The blood glucose can be measured in one minute.

Table 1 The clinical trial results

\begin{tabular}{cccc}
\hline & Parkers' error gird (A-E) & correlation & glucose range \\
\hline overall & $287(58.33 \%), 194(39.43 \%), 11(2.24 \%), 0(0 \%), 0(0 \%)$ & 0.6902 & $4.0-24.0 \mathrm{mmol} / \mathrm{L}$ \\
fasting & $174(70.45 \%), 68(27.53 \%), 5(2.02 \%), 0(0 \%), 0(0 \%)$ & 0.8394 & $4.0-17.3 \mathrm{mmol} / \mathrm{L}$ \\
postprandial & $113(46.12 \%), 126(51.43 \%), 6(2.45 \%), 0(0 \%), 0(0 \%)$ & 0.5393 & $4.8-24.0 \mathrm{mmol} / \mathrm{L}$ \\
\hline
\end{tabular}

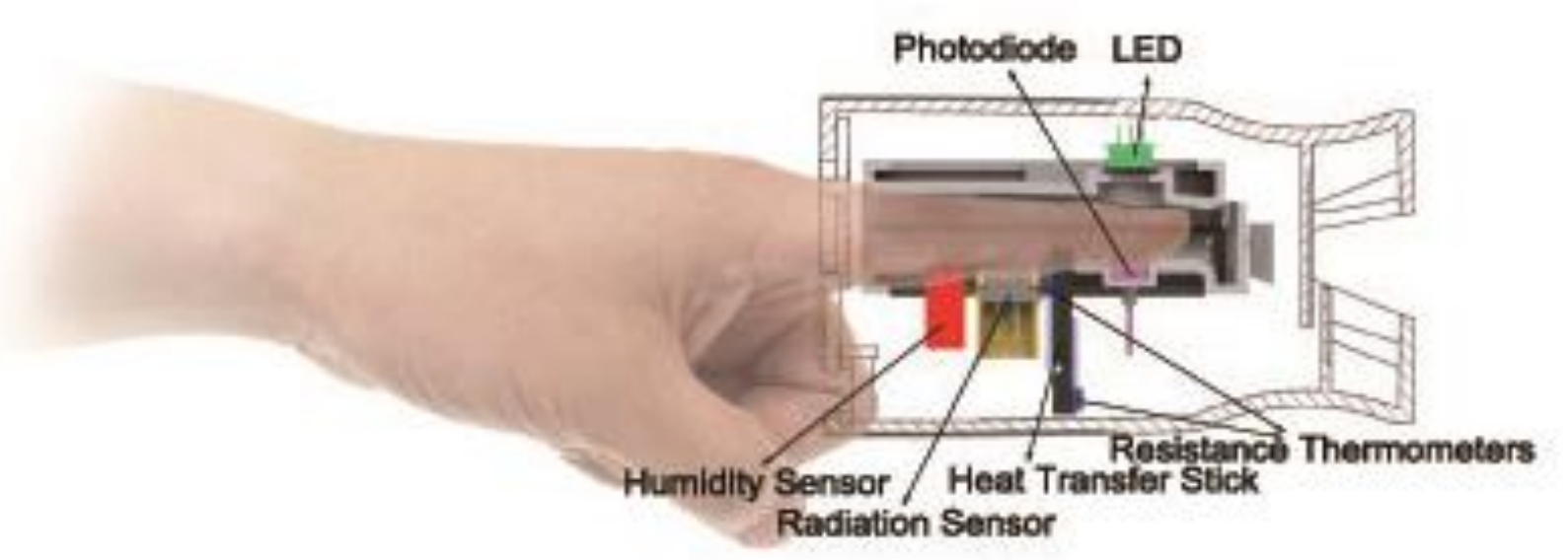

Figure 2 The probe structure
The Metabolic Heat Conformation Method: Blood glucose is the main material to participate in human metabolism. The metabolic heat is related to blood glucose concentration and oxygen consumption. When in the heat balance state, the metabolic rate is equal to the heat emitted from body to the environment. Thus, we can measure the glucose by measuring the metabolic related parameters such as environment temperature, environment humidity, body temperature, body humidity, blood flow, $\mathrm{SpO} 2$.

The Clinical Trial Design: The recruited type 2 diabetes subjects arrived at the test center before $8 \mathrm{am}$, then took a rest for 30 minutes to make sure the volunteers are in heat balance state with the environment. The fasting calibration was done first, then the noninvasive test was performed 20 minutes later. The fingertip blood and serum plasma glucose test were performed immediately after the noninvasive test, and the fasting glucose test was finished. The subjects then ate $80 \mathrm{~g}$ noodles in 15 minutes. Then in 2 hours postprandial, the noninvasive test, fingertip blood test and serum plasma test were conducted in sequence. All the three tests were performed two times, the average of the two results was taken.

Results: A total of 254 type 2 diabetes subjects were enrolled, including 118 female and 136 male, with an average age of 59.3 years. Comparison of the noninvasive glucose results and the serum blood glucose result: overall glucose range was $4.0-23.8 \mathrm{mmol} / \mathrm{L}$, the correlation was 0.6902 , the RMSE (root-mean-square error) was 2.668 $\mathrm{mmol} / \mathrm{L}$; the fasting glucose range was $4.0-17.3 \mathrm{mmol} / \mathrm{L}$, the correlation was 0.8394 , the RMSE was $1.855 \mathrm{mmol} / \mathrm{L}$; the 2 hours postprandial glucose range was $4.7-23.8 \mathrm{mmol} / \mathrm{L}$, the correlation was 0 . 5393, the RMSE was $3.290 \mathrm{mmol} / \mathrm{L}$. The results in the Parkers' error grid were shown in Fig. 3 and Tab. 1. The fasting results were showed in black and the postprandial results were showed in red.

Discussion: The noninvasive blood glucose monitor based on metabolic heat conformation method showed a good correlation with the serum glucose in fasting state, while in postprandial state the correlation reduced. This may related to the greater variability of the parameters of local circulation state, temperature and humidity in postprandial state. The method requires the user and the environment are in heat balance state, which is not easy to quantify. We need to make a trade-off between test convenience and accuracy, rest in the environment for 20 to 30 minutes is a suitable choice. It is suggested that, with a further technical improvement or more specific use conditions, the noninvasive blood glucose monitor based on metabolic heat conformation method have a great prospect in noninvasive glucose monitoring in clinical use.

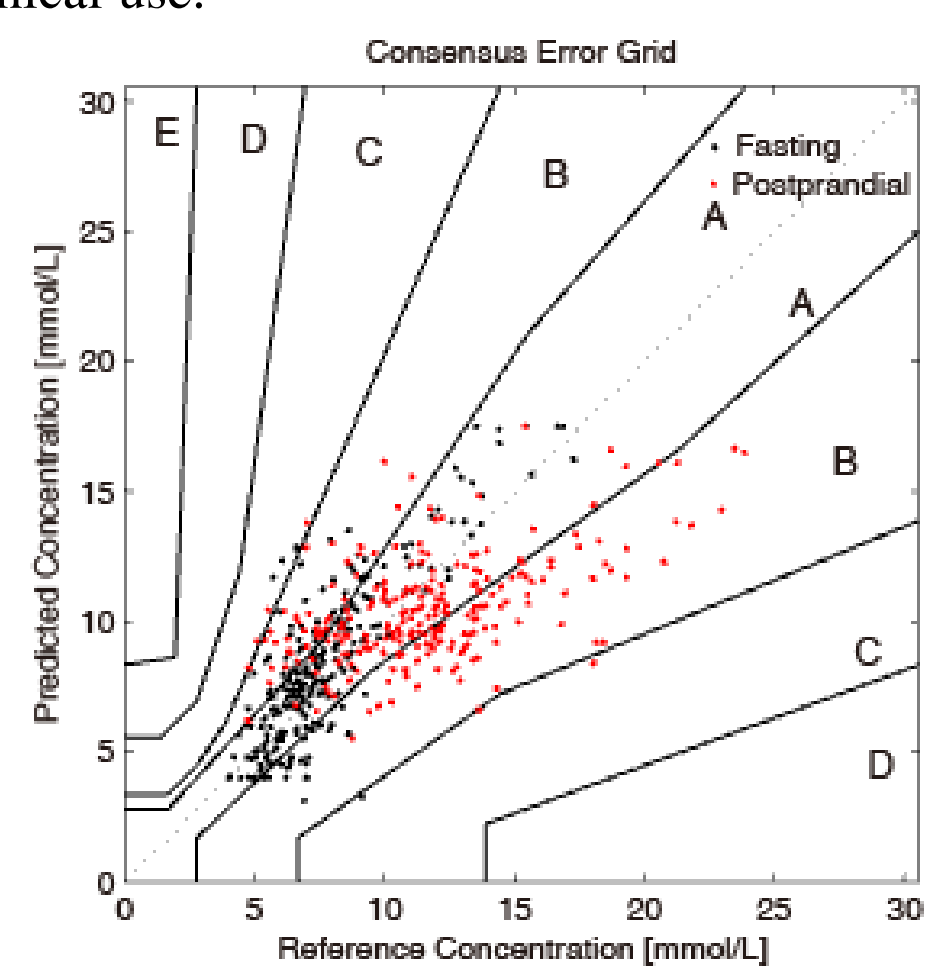

Figure 3 The Parkes' error grid result 